Review

\title{
Refractive Index Measurement of Liquids Based on Microstructured Optical Fibers
}

\section{Susana Silva ${ }^{1, \dagger}$, Paulo Roriz ${ }^{1,2, \dagger}$ and Orlando Frazão ${ }^{1,3, \dagger, *}$}

1 INESC Porto, Rua do Campo Alegre 687, 4169-007 Porto, Portugal;

E-Mails: sfsilva@inescporto.pt (S.S.); pjro@inescporto.pt (P.R.)

2 ISMAI-Instituto Universitário da Maia, Av. Carlos Oliveira Campos-Castelo da Maia, 4475-690 Maia, Portugal

3 Departamento de Física e Astronomia da Faculdade de Ciências, Universidade do Porto, Rua do Campo Alegre 687, 4169-007 Porto, Portugal

$\dagger$ These authors contributed equally to this work.

* Author to whom correspondence should be addressed; E-Mail: ofrazao@inescporto.pt; Tel.: +351-220-402-301; Fax: +351-220-402-734.

Received: 31 October 2014; in revised form: 3 December 2014 / Accepted: 3 December 2014 / Published: 8 December 2014

\begin{abstract}
This review is focused on microstructured optical fiber sensors developed in recent years for liquid RI sensing. The review is divided into three parts: the first section introduces a general view of the most relevant refractometric sensors that have been reported over the last thirty years. Section 2 discusses several microstructured optical fiber designs, namely, suspended-core fiber, photonic crystal fiber, large-core air-clad photonic crystal fiber, and others. This part is also divided into two main groups: the interferometric-based and resonance-based configurations. The sensing methods rely either on full/selective filling of the microstructured fiber air holes with a liquid analyte or by simply immersing the sensing fiber into the liquid analyte. The sensitivities and resolutions are tabled at the end of this section followed by a brief discussion of the obtained results. The last section concludes with some remarks about the microstructured fiber-based configurations developed for RI sensing and their potential for future applications.
\end{abstract}

Keywords: optical fiber sensors; microstructured fibers; refractive index 


\section{Introduction}

The great importance of measuring the refractive index (RI) in order to characterize the optical properties of fluids has led, over the past decades, to the development of RI sensors for applications in several areas such as the measurement of salinity of water [1], fuel quality analysis [2], or biotechnology processes [3]. In addition, direct measurements of RI were shown to provide valuable information on drug/DNA interaction and cell growth [4,5].

The Abbe refractometer is a well-known standard device that measures RI [6,7]; however, limitations of traditional bulk refractometers as size and weight have urged the development of alternative sensors. An active field of research has currently focused on optical fibers as an alternative device for RI measurement. Optical fiber-based RI sensors have found numerous applications in environmental, chemical and biological sensing [8]; they may indeed be rather compact in size, and can also be made suitable for distributed or tip-based sensing.

In fact, several distinct schemes for RI sensing based on optical fibers have been described over the last thirty years. In 1983, Cooper et al. [9] proposed the first optical fiber refractometer to measure RI in several liquids. This configuration combined a bulk refractometric system with a cladding-stripped optical fiber to study the RI behavior of liquids at the infrared (IR) range. Later, in 1984, Kumar et al. [10] presented one of the first refractometers based in a tapered optical fiber. The fiber device used a multimode plastic-clad silica core fiber and in a small section the cladding was removed and tapered by electrical spark-heating. The optical power in the output was dependent on the RI surrounding medium of the fiber taper.

The feasibility of a simple fiber optic technique, based on Fresnel reflection from the fiber tip, was demonstrated in 1987 by Meyer et al. [11]-by measuring retro-reflection from the interface between the core of a single-mode fiber and the liquid sample, for various RI. Several years later, Kim et al. [12] demonstrated the concept with an optical fiber coupler, in which one of the fiber arms was used as probe, and the other as reference. The RI of a few liquids was then successfully measured, by determining the ratio of reflected signals from the fiber-air and fiber-liquids interfaces.

In a different line of research, Jorgenson et al. [13] presented in 1993 an optical fiber sensor that utilized surface plasmon resonance (SPR) excitation. The sensing element was in this case a section of the fiber from which the cladding was removed-and a thin layer of highly reflecting metal was symmetrically deposited onto the fiber core, thus providing high sensitivity the RI changes of the external medium.

A long-period grating (LPG)-based RI sensor was produced in 1996 by Bhatia et al. [14]-the sensitivity to external perturbations came from cladding mode interactions with the surrounding medium.

Asseh et al. [15] developed in 1998 an evanescent field RI sensor based on a fiber Bragg grating (FBG) with etched cladding, interrogated by a tunable DBR laser. This type of RI sensor relied on the evanescent tail of the core modes under fiber etching conditions. In addition, when in contact with the surrounding medium, it was sensitive to RI variations.

In recent years, fiber-based RI sensors have relied on different approaches; for instance, Wang et al. [16] presented in 2006 a numerical analysis on multimode interference (MMI) effects. The sensing head relied in a singlemode-multimode-singlemode (SMS) structure where the surrounding liquid sample worked as the cladding medium. In 2008, Silva et al. [17] reported a fiber Fabry-Perot 
(FP) interferometer; this sensor relied on the combination of a short FBG with Fresnel reflection at the fiber tip, and the RI information was derived from the fringe visibility variation of the interferometer.

The appearance of new fiber geometries and drawing techniques allowed the fabrication of microstructured optical fibers (MOFs). These fibers have shown to be highly promising for sensing applications, due to its unique guiding properties, having greatly contributed to the expansion of RI sensing-area of research. The interaction between the evanescent field in microstructured fiber holes and the external medium (gaseous/liquid analyte) has been the object of extensive study for sensing purposes which has resulted several published works [18-22].

This review focuses on MOF sensors developed in recent years (since 2008) for liquid RI sensing. Section 1 introduces a general view of the most relevant refractometric sensors that have been reported over the last thirty years. Following that, Section 2 focuses specifically in MOF sensors for refractometric applications and it is divided in two main groups: the interferometric-based and resonance-based MOF configurations. The various types of MOF designs are presented, namely, the suspended-core fiber, photonic crystal fiber (PCF), large-core air-clad PCF, and SPR in PCFs. The configurations, sensitivities and resolutions are tabled at the end of this section followed by a brief discussion of the obtained results. Section 3 concludes with some remarks about the MOF configurations developed for RI sensing and their potential for future applications.

\section{Overview of Refractometer-Based Microstructured Fibers}

This section presents an overview of refractometer-based MOF sensors for liquid RI sensing. Two distinct groups are discussed: the interferometric-based and resonance-based MOF configurations. The first group relies on FP interferometry, Mach-Zehnder (MZ) interferometry, intermodal and multimodal interference, and using MOF-based configurations. The second group discusses configurations based on the combination of MOFs with FBGs, LPGs, SPR and other types of evanescent-wave devices. At the end of this section, a table with the results obtained with such sensors is presented.

\subsection{Interferometric-Based MOF Configurations}

In 2008, Jha et al. [23,24] proposed a compact in reflection modal interferometer consisting of a $24 \mathrm{~mm}$-long large-mode area photonic crystal fiber (PCF) spliced to a standard singlemode fiber (SMF), thus forming a sensing probe. In the splice, the voids of the PCF were fully collapsed allowing coupling and recombining PCF core and cladding modes. The detail of the sensing head is shown in Figure 1. The RI measurement relied on depositing a single drop of oil on the PCF surface near the splice region and avoiding at the same time the infiltration of sample into the PCF voids. The propagation constant of the cladding modes could be modified with the use of oils with different RIs. The interferometer was used for RI measurements in the 1.33-1.44 range: at the high index range of $1.38-1.44$, a maximum resolution of $2.9 \times 10^{-4}$ RIU was found; while at indices in the 1.33-1.38 range the resolution was $2 \times 10^{-3}$ RIU.

Later, Rao et al. [25] developed a refractometer based on an intrinsic fiber FP interferometer. The fiber tip was formed by a section of an endlessly singlemode PCF spliced between two standard SMFs, as depicted in Figure 2. The sensing element (2.34 mm-long PCF) was then sandwiched between two 
FP mirrors which originated an interference pattern that is sensitive to the RI variation of the external medium. In this case, the sensing head was fully immersed in a series of mixtures consisting of volume concentrations of glycerin in water. The RI change of glycerin solutions was measured by changes of fringe visibility of the optical spectrum-a sensitivity of 4.59/RI over the range from 1.32 to 1.44 was achieved as well as a resolution of $2 \times 10^{-5}$ RIU.

Figure 1. Sensing head designed for refractive index (RI) sensing using a fiber probe based on a large-mode area photonic crystal fiber (PCF) [23,24] and detail of the PCF cross-section view.

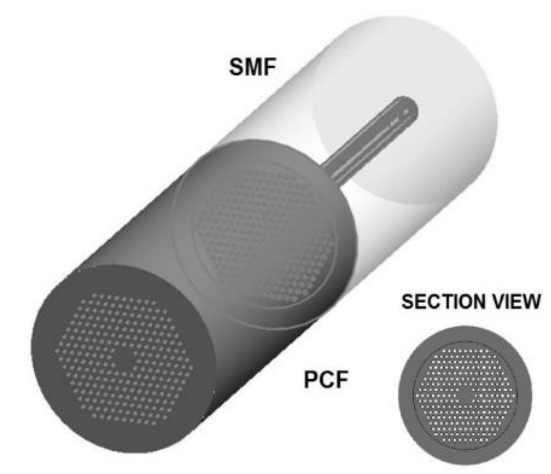

Figure 2. Refractometric fiber Fabry-Perot tip based on an endlessly singlemode PCF [25].

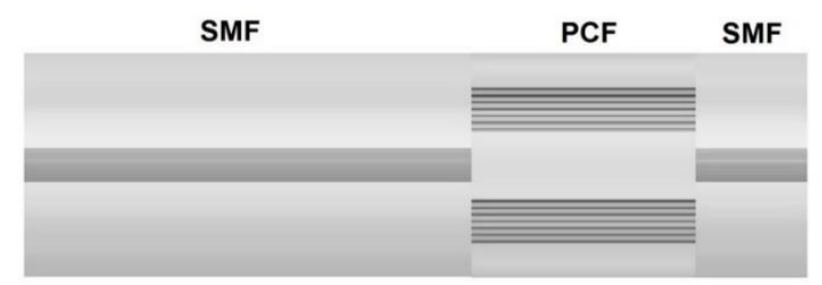

In 2009, Frazão et al. [26] presented an FP tip sensor based on a suspended-core fiber. The FP cavities were formed using a small section of the suspended-core fiber $(210 \mu \mathrm{m})$ spliced between SMFs, with the last section of the SMF being cleaved and presenting sensitivity to RI changes of the external medium. Figure 3 presents the detail of the developed sensing head. The FP tip sensor was fully immersed in liquids with different RIs within the range of 1.332-1.427. The measurement of RI changes was performed by fringe visibility variation and also through the analysis of the fast Fourier transform (FFT). Using the first method, a sensitivity of $-2.03 / \mathrm{RI}$ and resolution of $7 \times 10^{-4} \mathrm{RIU}$ was achieved; using the FFT analysis, better results were obtained by, namely, a sensitivity of $-11.27 /$ RIU and a resolution of $2 \times 10^{-4}$ RIU.

Wu et al. [27] presented a microfluidic RI sensor based on a directional coupler architecture using a solid-core PCF. The device sensor achieved very high sensitivity by coupling the core mode to a mode in the adjacent fluid-filled waveguide that was beyond modal cutoff, and with a strong field overlap. In this case, the principle of operation relied that any temperature induced changes in propagation properties can be attributed predominantly to changes in the RI of the fluid. Therefore, the fluid-filled PCF device (RI of 1.50 at $25{ }^{\circ} \mathrm{C}$ ) was submitted to $c a .52{ }^{\circ} \mathrm{C}$ and an RI sensitivity of $38,000 \mathrm{~nm} / \mathrm{RIU}$ was indeed achieved and a detection limit of $4.6 \times 10^{-7}$ RIU. This was the highest sensitivity obtained so far with PCF-based devices for RI sensing. 
Figure 3. Fabry-Perot tip sensor based on a PCF spliced between singlemode fiber (SMFs) [26] and detail of the PCF cross-section view.

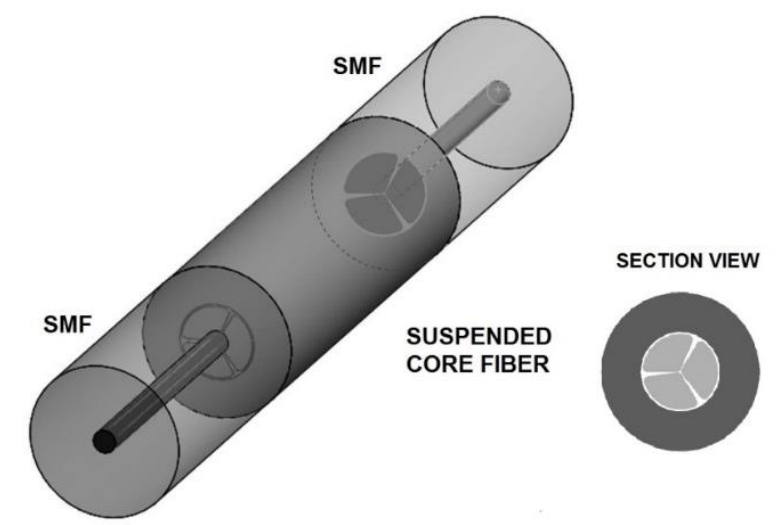

In 2010, Park et al. [28] constructed a PCF-based reflection type refractometer insensitive to temperature for RI measurement. The fiber structure was developed by collapsing the air-holes at a middle point of the PCF and splicing afterwards a $20 \mu \mathrm{m}$-long coreless silica fiber (with a gold-coating at its end), thus forming interference. The sensing probe was fully immersed in RI matching oils in the high RI range 1.410-1.430 and a sensitivity of $850 \mathrm{~nm} / \mathrm{RIU}$ was obtained.

In 2011, Gong et al. [29] demonstrated a miniature modal interferometer based on a hollow-core PCF for RI measurement. The device was fabricated by splicing a section of $1.2 \mathrm{~mm}$-long PCF between two SMFs and interrogated in transmission. The air holes of the PCF were fully collapsed by splicing procedure, originating a collapsed region of about $300 \mu \mathrm{m}$ in length, thus causing a modal interferometer. The transmission spectrum of the PCF sensor immersed in an aqueous analyte was measured for different RIs. Resolutions of $8.1 \times 10^{-4}$ RIU in the range $1.35-1.39$, and $4.3 \times 10^{-4}$ RIU in the range 1.39-1.43 were achieved. Sensitivities of $24.5 \mathrm{~nm} / \mathrm{RIU}$ and $46.5 \mathrm{~nm} / \mathrm{RIU}$ for the same RI ranges, respectively, were also obtained.

From a different perspective, Silva et al. [30] developed a refractometer based on multimodal interference, which relied on a large-core air-clad PCF spliced between two SMFs and interrogated in transmission. The detail of the sensing fiber is presented in Figure 4. Using two distinct large-core air-clad PCF geometries - one for RI measurement and the other for temperature compensation-it was possible to implement a device sensitive to RI changes in water induced by temperature variations. For the RI range of 1.3196-1.3171, results indicated a maximum sensitivity of $800 \mathrm{~nm} / \mathrm{RIU}$ and a resolution of $3.4 \times 10^{-5}$ RIU.

Sun et al. [31] proposed instead a dual-core PCF sensor based on a conventional solid core and a microstructured core. The sensing principle relied on selectively resonant coupling between both solid and microstructured cores. The microstructured core was composed of several air-holes that were filled with a low index analyte. Numerical simulation has shown that a detection limit of $2.02 \times 10^{-6}$ RIU and a sensitivity of $8500 \mathrm{~nm} / \mathrm{RIU}$ could be achieved for the analyte with an RI of 1.33. In the same line of research, Lee et al. [32] reported the experimental work and theoretical analysis of a MOF sensor consisting of a central Ge-doped silica core with a parallel hole in silica cladding. Intermodal interference was observed between three specific modes $\left(\mathrm{HE}_{21}, \mathrm{TM}_{01}\right.$ and $\left.\mathrm{TE}_{01}\right)$ of the liquid-filled hollow-core and the Ge-doped silica core. Experimentally, the hollow-core was filled with fluids of RI 
between 1.5 and 1.66 and sensitivities of $3183 \mathrm{~nm} / \mathrm{RIU}$ (TE01), $3259 \mathrm{~nm} / \mathrm{RIU}$ (TM01) and $2956 \mathrm{~nm} / \mathrm{RIU}$ $\left(\mathrm{HE}_{21}\right)$ were achieved.

Figure 4. Sensing head designed for RI sensing using a large-core air-clad PCF spliced between two SMFs [30] and detail of the PCF cross-section view.

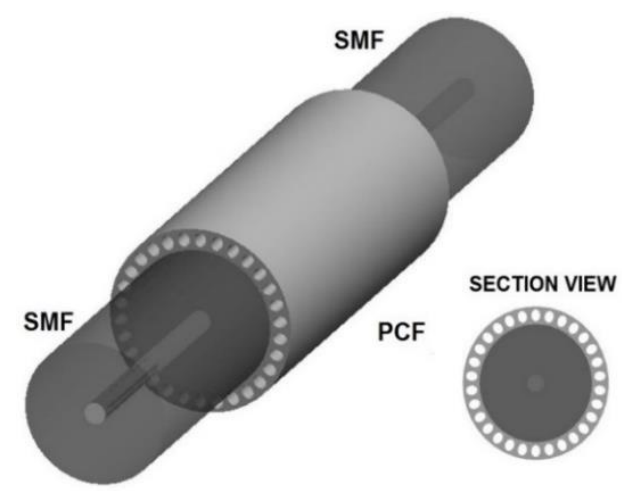

In 2012, Qian et al. [33] presented an intensity-based refractometric sensor that relied on the combination of an intermodal PCF interferometer as a sensing head and an FBG as a demodulating element. A short section of PCF $(2.5 \mathrm{~cm})$ was spliced between two SMFs and the air-holes in the splice regions were fully collapsed in order to form the intermodal PCF interferometer. An FBG was connected in series after the PCF interferometer which was used to reflect the optical power at the Bragg wavelength of the interference transmission spectrum. By immersing the PCF sensor tip in different external RI liquids, the corresponding reflective power of the FBG changed, which resulted from the shift on the interference spectrum of the intermodal PCF interferometer. The RI sensitivity was as low as $70 \mu \mathrm{W} / \mathrm{RIU}$ at the RI of 1.340 and increased for $679 \mathrm{~W} / \mathrm{RIU}$ at the RI of 1.360 . A maximum resolution of $1.5 \times 10^{-5} \mathrm{RIU}$ was also attained.

Wong et al. [34] proposed instead a PCF-based Mach-Zehnder interferometer (MZI) by splicing a short section of PCF between two SMFs and collapsing the air holes over a short region at the two splicing points. The PCF-MZI was formed due to the coupling between core and cladding modes in the collapsed region. The sensor was immersed in liquids with RIs within the range of 1.3515-1.360; when using the conventional OSA interrogation, a sensitivity of $101.25 \mathrm{~nm} / \mathrm{RIU}$ and resolution of $2.9 \times 10^{-4}$ RIU could be achieved. By placing the sensor inside a cavity ring-down loop, an improved minimum detectable RI of $7.8 \times 10^{-5}$ RIU was obtained.

Recently, Moura et al. [35] proposed a sensing configuration for distinct fluid evaporation monitoring using a suspended-core fiber tip. Figure 5 shows the detail of the sensing head used in the experiment. A $0.34 \mathrm{~mm}$-long suspended-core fiber was to an SMF in order to form the sensing tip. Strong differences between the evaporation processes of acetone and isopropyl alcohol (IPA) were observed, both in terms of the signal's intensity fluctuations and total duration. In each fluid, the main signal variations were due to changes in reflectivity inside a collapsed region of the suspended-core fiber near the spliced interface with the SMF and caused by the effective RI variation inside the suspended-core fiber. In this experiment it was observed that acetone and IPA evaporation took an average time of 4.1 and $6.2 \mathrm{~s}$, respectively. 
Figure 5. Sensing head designed for RI sensing based a conventional suspended-core fiber in a fiber tip configuration [35] and detail of the suspended-core fiber cross-section view.

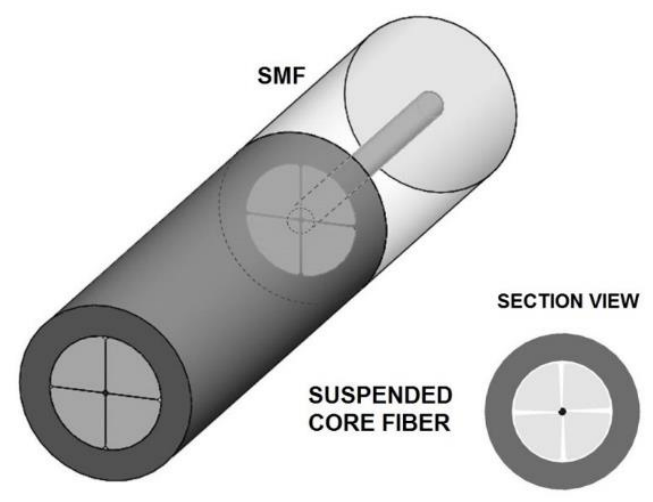

In the same line of research, Silva et al. [36] developed a multimodal interferometer based on a microstructured fiber tip for the detection of the evaporation process of acetone. The geometry consisted of a capillary tube, in which an offset Ge-doped core was fused, and spliced at the end of a standard SMF. The fiber tip sensor structure was immersed in liquid acetone and allowed monitoring in real time the evaporation process of acetone, due to RI variation of the external medium with increasing temperature-a short detection time of $\sim 1 \mathrm{~s}$ was achieved.

Wang et al. [37] presented a highly birefringent microfiber (up to $10^{-2}$ ) with a rhombus-like crosssectional shape. The microfiber was housed inside a photonic microcell made by locally heating and pressurizing selected air-holes of an endless single-mode PCF. The microcell was filled with a liquid $\mathrm{RI}$ at 1.3 and, in the temperature range of 25 to $95{ }^{\circ} \mathrm{C}$, exhibiting a high temperature sensitivity of $3 \mathrm{~nm} /{ }^{\circ} \mathrm{C}$, which corresponded to a RI sensitivity of $9.1 \times 10^{3} \mathrm{~nm} / \mathrm{RIU}$.

Torres et al. [38] reported a two-core chirped MOF for RI sensing of fluids. It was shown that by introducing a chirp in the hole size, the MOF could act as a structure with decoupled cores, thus forming a Mach-Zehnder interferometer in which the analyte directly modulated the device transmittance by its differential influence on the RI of each core mode. The sensing structure was submitted to analytes within the RI range of 1.33-1.44; a high RI sensitivity could be achieved by transmittance changes of 300/RIU at an RI of 1.42, and a detection limit of $3 \times 10^{-6}$ RIU.

\subsection{Resonance-Based MOF Configurations}

In 2008, Hassani et al. [39] reported SPR sensors based on the planar photonic crystal waveguide, solid-, and analyte-filled core Bragg fibers and a solid-core honeycomb PCF. The waveguide and Bragg fibers were gold-coated and, in the case of the PCF, the honeycomb air channels were gold-plated for plasmon excitation. Each sensor was immersed in an aqueous analyte with an RI of 1.33. The best results were found for the solid-core Bragg fiber which presented a sensitivity as high as $12000 \mathrm{~nm} / \mathrm{RIU}$ and a sensor resolution as low as $8.3 \times 10^{-6} \mathrm{RIU}$. In the case of the SPR-based PCF sensor, a maximum sensitivity of $2800 \mathrm{~nm} / \mathrm{RIU}$ and resolution of $3.6 \times 10^{-5} \mathrm{RIU}$ was attained.

Rindorf and Bang [40] demonstrated a highly sensitive refractometer based on a LPG written in a large-mode area PCF. The PCF-LPG was immersed in methanol in order to fill the air holes. The principle of operation relied on RI changes of methanol with increasing temperature. A maximum 
sensitivity of $1500 \mathrm{~nm} / \mathrm{RIU}$ at the RI of 1.33 was achieved, with a minimum detectable RI change of $2 \times 10^{-5}$ RIU.

Following the same line of research, He et al. [41] reported a study of LPGs inscribed in endlessly singlemode PCFs filled with air or water using a $\mathrm{CO}_{2}$ laser scanner. The cladding air channels of both sensors were filled with $\mathrm{NaCl}$ aqueous solutions at various concentrations. The LPG sensor fabricated in water exhibited an RI resolution of $4.42 \times 10^{-7}$ RIU over the RI range of 1.33-1.35; while the one inscribed in a PCF filled with air presented a slightly smaller resolution of $3.34 \times 10^{-7}$ RIU over the RI range of 1.33-1.34. The observed difference was caused by the narrower full width at half maximum of the resonance spectrum obtained by the LPG written in the PCF filled with water.

In 2010, Yu et al. $[42,43]$ reported instead the numerical analysis of an SPR-based PCF sensor for RI measurement. The sensor consisted of selectively metal-coated air holes containing analyte channels, which enhanced the phase matching between the plasmonic mode and the core-guided mode. The PCF structure consisted of a central air hole and two rings of symmetric hexagonally arranged holes. To simulate SPR, half of the outer air holes were coated with gold. Numerical analysis at the RI range from 1.37 to 1.41 allowed obtaining a sensitivity of $5500 \mathrm{~nm} / \mathrm{RIU}$ and an estimated resolution of $10^{-6}$ RIU. Later, the same research group presented numerical analysis on a wagon wheel-based microstructured fiber as a multichannel plasmonic sensor for the measurement of RI [44]. The large air-holes, coated with gold layers, could facilitate sample loading, enabling dual analyte detection. Numerical results have shown that an average sensitivity of $6.5 \times 10^{-6}$ RIU for each channel could be achieved over a dynamic index range of 1.33 to 1.36 .

In 2011, Wong et al. [45] proposed a miniature PCF-RI sensor based on field mode excitation. The sensor was fabricated by melting one end of the PCF into a rounded tip, spliced and collapsed the other end with an SMF. The rounded tip was able to induce cladding mode excitation, which resulted in an additional phase delay. The PCF sensor was immersed into glucose solutions with different RI values; a linear response of $262.28 \mathrm{~nm} / \mathrm{RIU}$ in the RI range of 1.337 to 1.395 was obtained as well as a resolution of $3.8113 \times 10^{-5} \mathrm{RIU}$. The sensor was also shown to be insensitive to environmental temperature.

Zhang et al. [46] reported in turn a MOF with an inscribed FBG for microfluidic sensing applications. The MOF was specially designed to be with a large amount of microholes for fluid filling and a solid core where the FBG was written. Experimental results revealed that the sensitivity of the sensor depended on both measurement of RI range and the order of fiber modes. Therefore, in the RI range of 1.31-1.38, the Bragg wavelength of the fundamental mode was not sensitive to RI changes; however, in the RI range of 1.4-1.44, the Bragg wavelength exhibited sensitivities of the first threeorder modes of $15 \mathrm{~nm} / \mathrm{RIU}, 21 \mathrm{~nm} / \mathrm{RIU}$, and $45 \mathrm{~nm} / \mathrm{RIU}$, respectively, and a minimum detectable RI change of $2.2 \times 10^{-5}$ RIU to $6.7 \times 10^{-5}$ RIU depending on the order of fiber modes.

In 2012, Lu et al. [47] investigated numerically an SPR sensor based on a grapefruit PCF filled with different numbers of silver nanowires for RI sensing in aqueous environments. The simulation results showed that the intensity sensitivity was related to the number of nanowires placed inside the PCF and the distance between two nanowires. For the RI range $1.33-1.335$, a sensitivity of $2400 \mathrm{~nm} / \mathrm{RIU}$ was obtained, corresponding to a resolution of $4.51 \times 10^{-5}$ RIU.

In a similar line of research, Shuai et al. [48] presented a numerical study on a closed-form multi-core holey fiber based SPR sensor. The central air-core was gold-coated and served as the 
analyte channel-thus performing selective filling for RI sensing. It was found that not only phase matching but also loss matching played a key role in the coupling process between the fundamental mode and plasmonic mode. The coupling transformed from incomplete to complete coupling with increasing analyte RI. An average sensitivity of $2929.39 \mathrm{~nm} / \mathrm{RIU}$, in the RI range of $1.33-1.42$, and $9231.27 \mathrm{~nm} / \mathrm{RIU}$, in the high RI range of 1.43-1.53, was obtained.

In a different perspective, Li et al. [49] developed instead a tapered-PCF in transmission and results shown that RI sensitivity increased with decreasing taper waist diameter. For a taper waist of $30 \mu \mathrm{m}$ and in the RI range 1.33-1.34 a maximum sensitivity of $1629 \mathrm{~nm} / \mathrm{RIU}$ was attained.

In 2013, Guzmán-Sepúlveda et al. [50] developed a refractometer sensor based on a two-core fiber configuration. The sensing head was based on a $50 \mathrm{~mm}$-long section of a two-core fiber that was spliced between two SMFs and interrogated in transmission. The sensing fiber was also side-etched in order to enhance sensitivity of the evanescent interaction with the external medium. The fiber device was immersed in liquid with different RIs in the range from 1.3160 to 1.3943 and a sensitivity of $3119 \mathrm{~nm} / \mathrm{RIU}$ was attained. Most recently, Warren-Smith and Monro [51] reported FBGs written in exposed-core MOFs by femtosecond laser technique. The distal end of the fiber was then sealed with ultra-violet curable glue so that the entire sensor tip could be immersed in a liquid without penetrating the internal MOF holes. Due to the penetration of the guided field outside the fiber, the Bragg reflections were wavelength sensitive to the external RI-a sensitivity of only $1.1 \mathrm{~nm} / \mathrm{RIU}$ was obtained in the range 1.3-1.4.

An overview of the various types of refractometric MOF sensors developed for liquid RI sensing is presented in Table I, as well as the results obtained in terms of RI sensitivity and/or resolution. From Table I, one can observe that through the design it is possible to control the sensitivity using the wavelength shift or, in a few cases, the intensity variation. The configurations based on interference concept (references [23] to [38]) are easier to fabricate but present lower sensitivities when compared with the ones based on resonance concept. However, enhanced sensitivities may be achieved when selective filling of air holes is performed for RI sensing. The configurations based on resonance concept (references [39] to [51]) present better sensitivity results, namely the ones based on LPGs and SPR concept. The MOF configurations based on SPR are promising RI sensors for liquids as regarded by the numerical results reported so far. However, the proposed designs require selective filling of the fiber holes to obtain enhanced sensitivity to RI and, in practice, the air-holes of the structures are difficult to coat with the metal film. In overall, the average resolution of this type of structures is typically $10^{-5}$, usually limited by the interrogation system.

Table 1. Overview of the various types of MOF-based refractometers developed for liquid RI sensing and according RI sensitivity and/or resolution results.

\begin{tabular}{|c|c|c|c|c|}
\hline Structure Device & RI Range & RI Sensitivity & Resolution (RIU) & Reference \\
\hline Fiber tip & $1.38-1.44$ & & $2.9 \times 10^{-4}$ & \multirow{2}{*}[23,24]{} \\
\hline $\mathrm{SMF} /$ large-mode area $\mathrm{PCF}$ & $1.33-1.38$ & & $2 \times 10^{-3}$ & \\
\hline $\begin{array}{c}\text { Fabry-Perot fiber tip } \\
\text { SMF/endlessly singlemode PCF/SMF }\end{array}$ & $1.32-1.44$ & 4.59/RI & $2 \times 10^{-5}$ & [25] \\
\hline $\begin{array}{l}\text { Fabry-Perot fiber tip } \\
\text { SMF/ PCF/SMF }\end{array}$ & $1.332-1.427$ & $\begin{array}{c}-11.27 / \text { RIU } \\
\text { (Fast Fourier Transform) }\end{array}$ & $2 \times 10^{-4}$ & [26] \\
\hline $\begin{array}{l}\text { Directional coupler architecture using a } \\
\text { solid-core PCF }\end{array}$ & $1.50 @ 25^{\circ} \mathrm{C}$ & $\begin{array}{c}38,000 \mathrm{~nm} / \mathrm{RIU} \\
@ 52^{\circ} \mathrm{C}\end{array}$ & $4.6 \times 10^{-7}$ & [27] \\
\hline
\end{tabular}


Table 1. Cont.

\begin{tabular}{|c|c|c|c|c|}
\hline Structure Device & RI Range & RI Sensitivity & Resolution (RIU) & Reference \\
\hline $\begin{array}{l}\text { Interferometric fiber tip } \\
\text { PCF/coreless silica fiber }\end{array}$ & $1.410-1.430$ & $850 \mathrm{~nm} / \mathrm{RIU}$ & - & [28] \\
\hline SMF/Hollow-core PCF/SMF & $\begin{array}{l}1.35-1.39 \\
1.39-1.43\end{array}$ & $\begin{array}{l}24.5 \mathrm{~nm} / \mathrm{RIU} \\
46.5 \mathrm{~nm} / \mathrm{RIU}\end{array}$ & $\begin{array}{l}8.1 \times 10^{-4} \\
4.3 \times 10^{-4}\end{array}$ & [29] \\
\hline SMF/large-core air-clad PCF/SMF & $1.3196-1.3171$ & $800 \mathrm{~nm} / \mathrm{RIU}$ & $3.4 \times 10^{-5}$ & [30] \\
\hline $\begin{array}{c}\text { Dual-core PCF } \\
\text { (microstructured and solid cores) }\end{array}$ & 1.33 & $8500 \mathrm{~nm} / \mathrm{RIU}$ & $2.02 \times 10^{-6}$ & [31] \\
\hline $\begin{array}{c}\text { Dual-core MOF } \\
\text { (Ge-doped and side-hole cores) }\end{array}$ & $1.5-1.66$ & $\begin{array}{l}3259 \mathrm{~nm} / \mathrm{RIU}\left(\mathrm{TM}_{01}\right) \\
3183 \mathrm{~nm} / \mathrm{RIU}\left(\mathrm{TE}_{01}\right) \\
2956 \mathrm{~nm} / \mathrm{RIU}\left(\mathrm{HE}_{21}\right)\end{array}$ & - & [32] \\
\hline Intermodal PCF interferometer & $\begin{array}{l}1.340 \\
1.360\end{array}$ & $\begin{array}{l}70 \mu \mathrm{W} / \mathrm{RIU} \\
679 \mathrm{~W} / \mathrm{RIU}\end{array}$ & $1.5 \times 10^{-5}$ & [33] \\
\hline $\begin{array}{c}\text { Mach-Zehnder interferometer } \\
\text { SMF/PCF with collapsed air holes in } \\
\text { the splice region /SMF }\end{array}$ & $1.3515-1.360$ & $101.25 \mathrm{~nm} / \mathrm{RIU}$ & $2.9 \times 10^{-4}$ & [34] \\
\hline $\begin{array}{l}\text { Microcell based on an endless } \\
\text { single-mode PCF }\end{array}$ & 1.3 & $9100 \mathrm{~nm} / \mathrm{RIU}$ & - & [37] \\
\hline Two-core chirped MOF & 1.42 & 300/RIU & $3 \times 10^{-6}$ & [38] \\
\hline $\begin{array}{l}\text { SPR-based solid-core gold-coated } \\
\text { honeycomb PCF }\end{array}$ & 1.33 & $2800 \mathrm{~nm} / \mathrm{RIU}$ & $3.6 \times 10^{-5}$ & [39] \\
\hline LPG written in a large-mode area PCF & 1.33 & $1500 \mathrm{~nm} / \mathrm{RIU}$ & $2 \times 10^{-5}$ & [40] \\
\hline $\begin{array}{l}\text { LPG written in an endlessly } \\
\text { singlemode PCFfilled with water }\end{array}$ & $1.33-1.35$ & - & $4.42 \times 10^{-7}$ & [41] \\
\hline $\begin{array}{l}\text { SPR-based on a PCF with a central air } \\
\text { hole coated with gold }\end{array}$ & $1.37-1.41$ & $5500 \mathrm{~nm} / \mathrm{RIU}$ & $10^{-6}$ & {$[42,43]$} \\
\hline $\begin{array}{c}\text { SPR-based on a gold-coated wagon } \\
\text { wheel MOF }\end{array}$ & $1.33-1.36$ & - & $6.5 \times 10^{-6}$ & [44] \\
\hline Rounded tip-based PCF & $1.337-1.395$ & $262.28 \mathrm{~nm} / \mathrm{RIU}$ & - & [45] \\
\hline FBG written in a MOF & $1.4-1.44$ & $\begin{array}{l}15,21 \text { and } 45 \mathrm{~nm} / \mathrm{RIU} \\
\text { (first three-order modes) }\end{array}$ & 2.2 to $6.7 \times 10^{-5}$ & [46] \\
\hline $\begin{array}{l}\text { SPR-based grapefruit PCF filled with } \\
\text { different numbers of silver nanowires }\end{array}$ & $1.33-1.335$ & $2400 \mathrm{~nm} / \mathrm{RIU}$ & $4.51 \times 10^{-5}$ & [47] \\
\hline \multirow[t]{2}{*}{$\begin{array}{l}\text { SPR-based on a closed-form multi-core } \\
\text { holey fiber }\end{array}$} & $1.33-1.42$ & $2929.39 \mathrm{~nm} / \mathrm{RIU}$ & \multirow[t]{2}{*}{ - } & \multirow[t]{2}{*}{ [48] } \\
\hline & $1.43-1.53$ & $9231.27 \mathrm{~nm} / \mathrm{RIU}$ & & \\
\hline SMF/tapered-PCF/SMF & $1.33-1.34$ & $1629 \mathrm{~nm} / \mathrm{RIU}$ & - & [49] \\
\hline Etched two-core MOF & $1.3160-1.3943$ & $3119 \mathrm{~nm} / \mathrm{RIU}$ & - & {$[50]$} \\
\hline FBG written in exposed-core MOF & $1.3-1.4$ & $1.1 \mathrm{~nm} / \mathrm{RIU}$ & - & [51] \\
\hline
\end{tabular}

\section{Conclusions}

A review of refractive index measurement in liquids using microstructured fibers was presented. Several MOF designs was described, namely, suspended-core fibers, photonic crystal fibers, and large-core air-clad photonic crystal fibers. The reported sensing heads present a large variety of fiber designs and the sensing methods rely either on full/selective filling of the PCF air holes with a liquid analyte or by simply immersing the sensing fiber into the liquid analyte. From Table 1, it can be concluded that through the design it is possible to control the sensitivity using the wavelength shift or the intensity variation. The average resolution of this type of structures is typically $10^{-5}$. A promising RI sensing device is the one based on the combination of MOFs with thin films to generate SPR sensors. The PCF-based SPR sensors for liquid substances can be constructed by infiltrating the 
analyte into the metal-coated holes of the PCF. So far, numerical analysis of such SPR-PCF sensors has been reported. Although potentially presenting substantial increase of sensitivity to RI, the proposed designs require selective filling of the fiber holes to obtained enhanced sensitivity to RI. Moreover, in practice, the air-holes of the structures are difficult to coat with the metal film.

The future is promising in the RI area of research; however it is necessary to find the specific applications to make these into new commercial structures. One example is its application in a wider array of volatile compounds by identifying the main signal components in the evaporation response. This simple sensing setup could be used to distinguish different fluids and potentially recognize mixtures of volatile compounds. Refractometers have also applications in industrial and scientific laboratories, particularly in quality control settings where a rapid RI measurement indicates if the product is on specification. Some examples include salinity of water, sugar concentration of beverages and fermentation processes, hydrocarbon content of motor fuels and detection of proteins in solution.

\section{Acknowledgments}

This work was financed by the FCT, Fundação para a Ciência e Tecnologia (Portuguese Foundation for Science and Technology) and by the ERDF (European Regional Development Fund) through COMPETE Programme (Operational Programme for Competitiveness) within project FCOMP-010124-FEDER-037281; ON.2, O Novo Norte (Northern Portugal Regional Operational Programme), under the National Strategic Reference Framework, within project NORTE-07-0124-FEDER-000058; and S.S. received a Pos-Doc fellowship (ref. SFRH/BPD/92418/2013) also funded by FCT.

\section{Conflict of Interest}

The authors declare no conflict of interest.

\section{References}

1. Pereira, D.A.; Frazão, O.; Santos, J.L. Fibre Bragg grating sensing system for simultaneous measurement of salinity and temperature. Opt. Eng. 2004, 43, 299-304.

2. Osorio, J.H.; Mosquera, L.; Gouveia, C.J.; Biazoli, C.R.; Hayashi, J.G.; Jorge, P.A.S.; Cordeiro, C.M.B. High sensitivity LPG Mach-Zehnder sensor for real-time fuel conformity analysis. Meas. Sci. Technol. 2013, 24, 015102.

3. Jiménez-Márquez, F.; Vázquez, J.; Úbeda, J.; Sánchez-Rojas, J.L. Low-cost and portable refractive optoelectronic device for measuring wine fermentation kinetics. Sens. Actuators $B$ Chem. 2013, 178, 316-323.

4. Zibaii, M.I.; Kazemi, A.; Latifi, H.; Azar, M.K.; Hosseini, S.M.; Ghezelaiagh, M.H. Measuring bacterial growth by refractive index tapered fiber optic biosensor. J. Photochem. Photobiol. B Biol. 2010, 3, 313-320.

5. Zibaii, M.I.; Latifi, H.; Karami, M.; Gholami, M.; Hosseini, S.M.; Ghezelayagh, M.H. Non-adiabatic tapered optical fiber sensor for measuring the interaction between alpha-amino acids in aqueous carbohydrate solution. Meas. Sci. Technol. 2010, 21, 105801. 
6. Meeten, G.H.; North, A.N. Refractive index measurement of turbid colloidal fluids by transmission near the critical angle. Meas. Sci. Technol. 1991, 2, 441-447.

7. Rheims, J.; Koser, J.; Wriedt, T. Refractive-index measurements in the near-IR using an Abbe refractometer. Meas. Sci. Technol. 1997, 8, 601-605.

8. Jorge, P.A.S.; Silva, S.O.; Gouveia, C.; Tafulo, P.; Coelho, L.; Caldas, P.; Viegas, D.; Rego, G.; Baptista, J.M.; Santos, J.L.; et al. Fiber Optic-Based Refractive Index Sensing at INESC Porto. Sensors 2012, 12, 8371-8389.

9. Cooper, P.R. Refractive-index measurements of liquids used in conjunction with optical fibers. Appl. Opt. 1983, 22, 3070-3072.

10. Kumar, A.; Subrahmanyam, T.V.B.; Sharma, A.D.; Thyagarajan, K.; Pal, B.P.; Goyal, I.C. Novel refractometer using a tapered optical fiber. Electron. Lett. 1984, 20, 534-535.

11. Meyer, M.S.; Eesley, G.L. Optical fiber refractometer. Rev. Sci. Instrum. 1987, 58, 2047-2048.

12. Kim, C.-B.; Su, C.B. Measurement of the refractive index of liquids at 1.3 and 1.5 micron using a fiber optic Fresnel ratio meter. Meas. Sci. Technol. 2004, 15, 1683-1686.

13. Jorgenson, R.C.; Yee, S.S. A fiber-optic chemical sensor based on surface plasmon resonance. Sens. Actuators B Chem. 1993, 12, 213-220.

14. Bhatia, V.; Vengsarkar, A.M. Optical fiber long-period grating sensors. Opt. Lett. 1996, 21, 692-694.

15. Asseh, A.; Sandgren, S.; Ahlfeldt, H.; Sahlgren, B.; Edwall, G. Fiber optical Bragg refractometer. Fiber Integr. Opt. 1998, 17, 51-62.

16. Wang, Q.; Farrell, G. All-fiber multimode-interference based refractometer sensor: Proposal and design. Opt. Lett. 2006, 31, 317-319.

17. Silva, S.F.O.; Frazão, O.; Caldas, P.; Santos, J.L.; Araújo, F.M.; Ferreira, L.A. Optical fibre refractometer based on a Fabry-Pérot interferometer. Opt. Eng. 2008, 47, 054403.

18. Fini, J.M. Microstructure fibres for optical sensing in gases and liquids. Meas. Sci. Technol. 2004, $15,1120-1128$.

19. Cordeiro, C.M.B.; Franco, M.A.R.; Chesini, G.; Barretto, E.C.S.; Lwin, R.; Cruz, C.H.B.; Large, M.C.J. Microstructured-core optical fibre for evanescent sensing applications. Opt. Exp. 2006, 14, 13056-13066.

20. Troia, B.; Paolicelli, A.; Leonardis, F.D.; Passaro, V.M.N. Photonic Crystals for Optical Sensing: A Review. In Advances in Photonic Crystals; Vittorio, P., Ed.; InTech: Rijeka, Croatia, 2013, pp. 241-295.

21. Frazão, O.; Santos, J.; Araújo, F.; Ferreira, L. Optical sensing with photonic crystal fibers. Laser Photon. Rev. 2008, 2, 449-459.

22. Pinto, A.M.R.; Lopez-Amo, M. Photonic Crystal Fibers for Sensing Applications. J. Sens. 2012, 12, 598178.

23. Jha, R.; Villatoro, J.; Badenes, G. Ultrastable in reflection photonic crystal fiber modal interferometer for accurate refractive index sensing. Appl. Phys. Lett. 2008, 93, 191106.

24. Jha, R.; Villatoro, J.; Badenes, G.; Pruneri, V. Refractometry based on a photonic crystal fiber interferometer. Opt. Lett. 2009, 34, 617-619.

25. Rao, Y.-J.; Deng, M.; Duan, D.-W.; Zhu, T. In line fiber Fabry Perot refractive index tip sensor based on endlessly photonic crystal fiber. Sens. Actuators A Phys. 2008, 148, 33-38. 
26. Frazão, O.; Baptista, J.M.; Santos, J.L.; Kobelke, J.; Schuster, K. Refractive index tip sensor based on Fabry-Perot cavities formed by a suspended core fibre. JEOS:RP 2009, 4, 09041.

27. Wu, D.K.C.; Kuhlmey, B.T.; Eggleton, B.J. Ultrasensitive photonic crystal fiber refractive index sensor. Opt. Lett. 2009, 34, 322-324.

28. Park, K.S.; Choi, H.Y.; Park, S.J.; Paek, U.-C.; Lee, B.H. Temperature Robust Refractive Index Sensor Based on a Photonic Crystal Fiber Interferometer. IEEE Sens. J. 2010, 10, 1147-1148.

29. Gong, H.; Chan, C.C.; Zhang, Y.F.; Wong, W.C.; Dong, X. Miniature refractometer based on modal interference in a hollow-core photonic crystal fiber with collapsed splicing. J. Biomed. Opt. 2011, 16, 017004.

30. Silva, S.; Santos, J.L.; Malcata, F.X.; Kobelke, J.; Schuster, K.; Frazão, O. Optical Refractometer based on Large-Core Air-Clad Photonic Crystal fibers. Opt. Lett. 2011, 36, 852-854.

31. Sun, B.; Chen, M.-Y.; Zhang, Y.-K.; Yang, J.-C.; Yao, J.-Q.; Cui, H.-X. Microstructured-core photonic-crystal fiber for ultra-sensitive refractive index sensing. Opt. Express 2011, 19, 4091-4100.

32. Lee, H.W.; Schmidt, M.A.; Uebel, P.; Tyagi, H.; Joly, N.Y.; Scharrer, M.; Russell, P.S. Optofluidic refractive-index sensor in step-index fiber with parallel hollow micro-channel. Opt. Express 2011, 19, 8200-8007.

33. Qian, W.; Chan, C.C.; Zhao, C.-L.; Liu, Y.; Li, T.; Hu, L.; Ni, K.; Dong, X. Photonic crystal fiber refractive index sensor based on a fiber Bragg grating demodulation. Sens. Actuators B Chem. 2012, 166-167, 761-765.

34. Wong, W.C.; Zhou, W.; Chan, C.C.; Dong, X.; Leong, K.C. Cavity ringdown refractive index sensor using photonic crystal fiber interferometer. Sens. Actuators B Chem. 2012, 161, 108-113.

35. Moura, J.P.; Baierl, H.; Auguste, J.-L.; Jamier, R.; Roy, P.; Santos, J.L.; Frazão, O. Evaporation of volatile compounds in suspended-core fibers. Opt. Lett. 2014, 39, 3868-3871.

36. Silva, S.O.; Auguste, J.-L.; Jamier, R.; Rougier, S.; Baptista, J.M.; Santos, J.L.; Roy, P.; Frazão, O. Detection of Evaporation Process of Acetone with a Microstructured fibre in a Reflective Configuration. Opt. Eng. Lett. 2014, 53, 080501.

37. Wang, C.; Jin, W.; Liao, C.; Ma, J.; Jin, W.; Yang, F.; Ho, H.L.; Wang, Y. Highly birefringent suspended-core photonic microcells for refractive-index sensing. Appl. Phys. Lett. 2014, 105, 061105.

38. Torres, P.; Reyes-Vera, E.; Díez, A.; Andrés, M.V. Two-core transversally chirped microstructured optical fiber refractive index sensor. Opt. Lett. 2014, 39, 1593-1596.

39. Hassani, A.; Gauvreau, B.; Fehri, M.F.; Kabashin, A.; Skorobogatiy, M. Photonic Crystal Fiber and Waveguide-Based Surface Plasmon Resonance. Electromagnetics 2008, 28, 198-213.

40. Rindorf, L.; Bang, O. Highly sensitive refractometer with a photonic-crystal-fiber long-period grating. Opt. Lett. 2008, 33, 563-565.

41. He, Z.; Zhu, Y.; Du, H. Long-period gratings inscribed in air- and water-filled photonic crystal fiber for refractometric sensing of aqueous solution. Appl. Phys. Lett. 2008, 92, 044105.

42. Yu, X.; Zhang, Y.; Pan, S.; Shum, P.; Yan, M.; Leviatan, Y.; Li, C. A selectively coated photonic crystal fiber based surface plasmon resonance sensor. J. Opt. 2010, 12, 015005. 
43. Zhang, Y.; Xia, L.; Zhou, C.; Yu, X.; Liu, H.; Liu, D.; Zhang, Y. Microstructured fiber based plasmonic index sensor with optimized accuracy and calibration relation in large dynamic range. Opt. Commun. 2011, 284, 4161-4166.

44. Zhang, Y.; Zhou, C.; Xia, L.; Yu, X.; Liu, D. Wagon wheel fiber based multichannel plasmonic sensor. Opt. Express 2011, 19, 22863-22873.

45. Wong, W.C.; Chan, C.C.; Chen, L.H.; Tou, Z.Q.; Leong, K.C. Highly sensitive miniature photonic crystal fiber refractive index sensor based on mode field excitation. Opt. Lett. 2011, 36, 1731-1733.

46. Zhang, A.P.; Yan, G.; Gao, S.; He, S.; Kim, B.; Im, J.; Chung, Y. Microfluidic refractive-index sensors based on small-hole microstructured optical fiber Bragg gratings. Appl. Phys. Lett. 2011, 98, 221109.

47. Lu, Y.; Hao, C.-J.; Wu, B.-Q.; Huang, X.-H.; Wen, W.-Q.; Fu, X.-Y.; Yao, J.-Q. Grapefruit Fiber Filled with Silver Nanowires Surface Plasmon Resonance Sensor in Aqueous Environments. Sensors 2012, 12, 12016-12025.

48. Shuai, B.; Xia, L.; Zhang, Y.; Liu, D. A multi-core holey fiber based plasmonic sensor with large detection range and high linearity. Opt. Express 2012, 20, 5974-5986.

49. Li, C.; Qiu, S.-J.; Chen, Y.; Xu, F.; Lu, Y.-Q. Ultra-Sensitive Refractive Index Sensor with Slightly Tapered Photonic Crystal Fiber. IEEE Photon. Technol. Lett. 2012, 24, 1771-1774.

50. Guzmán-Sepúlveda, J.R.; Guzman-Cabrera, R.; Torres-Cisneros, M.; Sánchez-Mondragón, J.J.; May-Arrioja, D.A. A Highly Sensitive Fiber Optic Sensor Based on Two-Core Fiber for Refractive Index Measurement. Sensors 2013, 13, 14200-14213.

51. Warren-Smith, S.C.; Monro, T.M. Exposed core microstructured optical fiber Bragg gratings-refractive index sensing. Opt. Express 2014, 22, 1480-1489.

(C) 2014 by the authors; licensee MDPI, Basel, Switzerland. This article is an open access article distributed under the terms and conditions of the Creative Commons Attribution license (http://creativecommons.org/licenses/by/4.0/). 